CLINICAL STUDY

\title{
The association of serum adipocyte fatty acid-binding protein with coronary artery disease in Korean adults
}

\author{
E J Rhee ${ }^{1}$, W Y Lee ${ }^{1}$, C Y Park ${ }^{1}, \mathrm{~K}$ W Oh${ }^{1}$, B J Kim² ${ }^{2}$ K C Sung ${ }^{2}$ and B S Kim ${ }^{2}$ \\ ${ }^{1}$ Department of Endocrinology and Metabolism and ${ }^{2}$ Department of Cardiology, Kangbuk Samsung Hospital, Sungkyunkwan University School of Medicine, \\ 110746 Seoul, South Korea
}

(Correspondence should be addressed to W-Y Lee who is now at Division of Endocrinology and Metabolism, Department of Internal Medicine, Kangbuk Samsung Hospital, Sungkyunkwan University School of Medicine, no. 108 Pyung-Dong, Jongro-Ku, Seoul 110-746, South Korea;

Email: drlwy@hanmail.net)

\begin{abstract}
Objectives: Adipocyte fatty acid-binding protein (A-FABP), also known as aP2 or FABP4, is abundantly expressed in adipocytes and plays a role in glucose homeostasis. We analyzed the relationship between the coronary artery disease and serum FABP4 levels in Korean adults.

Methods: In a total of 234 Korean adults, in whom coronary angiograms were performed, anthropometric measurements were done and fasting glucose and lipid profiles were measured. Serum FABP4 levels were measured using ELISA. The presence of metabolic syndrome was diagnosed according to American Heart Association/National Heart, Lung and Blood Institute (AHA/NHBL) criteria with body mass index (BMI) substituted for waist circumference.

Results: Among the subjects, $31.6 \%$ had diabetes, $46.9 \%$ had metabolic syndrome, and mean log (FABP4) levels showed significantly higher levels in subjects with diabetes. Among the subjects, $42.4 \%$ had normal coronary vessel, $34.6 \%$ had 1-vessel disease, $13.7 \%$ had 2 -vessel disease, and $9.4 \%$ had 3-vessel disease. Among the parameters, mean age, fasting glucose, and log (FABP4) levels increased significantly as the numbers of stenotic vessel increased from normal to 3-vessel disease, and for FABP4, these significances showed a consistent trend for difference after adjustment for age, gender, $\mathrm{BMI}$, and fasting glucose $(P=0.072)$. Mean log $(\mathrm{FABP} 4)$ level showed lower values in subjects taking aspirin, and higher values in subjects taking statin and anti-hypertensive drugs.

Conclusions: Serum FABP4 levels increased as the numbers of stenotic coronary artery increased, although these differences were attenuated after adjustment for age and fasting glucose levels. Various anti-atherogenic medications showed different effects on the serum FABP4 levels, which need further investigation.
\end{abstract}

European Journal of Endocrinology 160 165-172

\section{Introduction}

Obesity and cardiovascular disease are two most prevalent diseases in the elderly $(1,2)$. Recent works focus on uncovering the link between these diseases and the common pathophysiological mechanisms providing the association between the two diseases. The more prevalent rate of atherosclerotic diseases in obese populations compared with lean subjects confirm the role of obesity in the development of atherosclerosis (3). The most simple explanation for the linkage between these two diseases is metabolic syndrome. Metabolic syndrome is a cluster of risk factors such as abdominal obesity, hyperglycemia, hypertension, and dyslipidemia, all of which is thought to cause insulin resistance due to visceral obesity (4). The seriousness of metabolic syndrome lies in the increased development of cardiovascular disease and type 2 diabete mellitus in subjects with this syndrome. Recent works report the dynamic function of adipose tissue as an endocrine organ, not only for storing the remnant energy but also for releasing various cytokines, adipokines and inflammatory markers, which contribute to the development of insulin resistance and atherosclerosis (5).

An important molecular pathway that integrates metabolic and inflammatory responses, and the subsequent atherosclerosis, involves the fatty acid-binding proteins (FABPs) that are commonly present in adipocyte and macrophages (6). FABPs are a family of small cytoplasmic proteins, 14-15 kDa, conserved through evolution from Drosophila to humans (7). There are nine types of FABPs, exhibiting a tissuespecific expression pattern, and often their abundance is regulated by the demands of the cells in which they are present (8). FABPs act as cytoplasmic lipid chaperones and play a role in the cellular trafficking of fatty acids 
and other lipid signals through their interaction with functional targets. Among them, adipocyte FABP (A-FABP), also known as aP2 or FABP4, is one of the most abundant protein in adipocytes and macrophages. The precise pathophysiological role of these small proteins has been recognized only upon the development of genetic models to examine their function in mice (9-11). In these experimental models, deficiency of FABP4 results in lowered triglycerides, increased insulin sensitivity when made obese, and protection against atherosclerosis in models of hypercholesterolemia. The impact of FABP4 deficiency on atherosclerosis is predominantly the result of its action in macrophages (12), and in a very recent study using selective deletion of FABPs in adipocyte and macrophage, and bonemarrow transplantation experiments in mice, Furuhashi et al. (13) demonstrated that neither macrophages nor adipocytes individually could account for the total impact of FABPs on systemic metabolism and suggest that interactions between these two cell types, particularly in adipose tissue, are critical for the inflammatory basis of metabolic deterioration.

It has been shown that FABP4 levels could be measured in human serum, and could be useful in clinical diagnosis of obesity-related metabolic syndrome (14). In the studies performed in non-diabetic Hong Kong subjects, high baseline serum FABP4 was predictive of metabolic syndrome and type 2 diabetes mellitus, independent of obesity and insulin resistance $(15,16)$. As the clinical importance of type 2 diabetes and metabolic syndrome is the development of cardiovascular disease, the clinical implications of FABPs have been studies in relation with atherosclerosis. Furuhashi et al. (13) demonstrated that an FABP4 inhibitor administration in $\mathrm{ApoE}^{-/-}$mouse model showed marked reductions in atherosclerotic lesion area compared with the vehicle-treated group, suggesting the effect of FABP4 inhibition might prevent not only diabetes or metabolic disease but also atherosclerosis. In a genetic study done by Tuncman et al. (17), individuals with the FAPB4/aP2 variant had lower serum triglyceride levels, exhibited reduced risk of cardiovascular disease, and lower expression level of adipose tissue FABP4. In a recent work by Yeung et al. (18), serum FABP4 levels were independently associated with carotid atherosclerosis in Chinese women. These findings suggest the role of FABP4 in metabolic syndrome and cardiovascular disease, although the precise role has not been clarified.

Although a few studies have been performed in relation to the association of FABP 4 and atherosclerosis, there are no studies performed on the direct relationship of serum FABP4 levels with atherosclerosis in human. Therefore, we designed this study to see the relationship between serum FABP4 levels and coronary artery disease (CAD) in subjects in whom coronary angiograms were performed due to chest pain.

\section{Subjects and methods}

\section{Study subjects}

The study was performed in 234 subjects in whom coronary angiograms were performed in the department of cardiology in Kangbuk Samsung Hospital, Sungkyunkwan University School of Medicine, Seoul, Korea, due to chest pain from May to September, 2003. The patients included 137 (58.5\%) men and 97 (41.5\%) women whose mean age was 58.2 years (range 26-87 years). Those with medical illnesses such as acute infection, chronic renal failure (serum creatini$\mathrm{ne} \geq 2.0 \mathrm{mg} / \mathrm{dl}$ ), malignancies, and other severe medical illnesses were excluded from the enrollment. The histories for smoking and medication of the participants, at the time of coronary angiograms, were obtained by the chart review and from the self-questionnaire.

Written informed consent was obtained from each participant and the study protocol conforms to the ethical guidelines of the 1975 Declaration of Helsinki as reflected in a priori approval by the institution's human research committee.

\section{Anthropometric measurements and blood chemistry}

Height, weight, systolic, and diastolic blood pressures were measured in duplicate and the results were averaged. Weight and height were measured in $\mathrm{kg}$ and $\mathrm{cm}$ respectively, down to two decimal points. The body mass index (BMI) was calculated by dividing the weight (kg) with the square of height (m). After 12 hours of fasting, blood sampling was done. Fasting blood glucose, total cholesterol, triglyceride, high-density lipoprotein cholesterol (HDL-C), and low-density lipoprotein cholesterol (LDL-C) were measured from the samples and the hexokinase method was used to measure blood glucose levels and enzymatic colorimetric test was used to measure the total cholesterol and triglyceride levels. The selective inhibition method was used to measure the level of HDL-C and the homogeneous enzymatic colorimetric test was used to measure the level of LDL-C.

\section{Measurements of serum FABP4 levels}

Blood samples were taken after an overnight fast and before the coronary angiogram. The serum was separated and stored at $-80^{\circ} \mathrm{C}$, and serum FABP4 levels were measured simultaneously later. The measurement was performed by an ELISA method (BioVendor Laboratory Medicine, Modrice, Czech Republic).

\section{Diagnosis of diabetes mellitus and metabolic syndrome}

The presence of diabetes mellitus was confirmed according to the criteria from the American Diabetes 
Association (19), or from the medical history of being treated with diabetes mellitus.

Based on the American Heart Association/National Heart, Lung, and Blood Institute (AHA/NHLBI) criteria with the BMI $\left(\geq 25 \mathrm{~kg} / \mathrm{m}^{2}\right)$ substituted for waist circumference, the diagnosis of MetS was made when the subject satisfied more than three categories among the five categories described below $(20,21)$;

1. Obesity: $\mathrm{BMI} \geq 25 \mathrm{~kg} / \mathrm{m}^{2}$

2. Hypertriglyceridemia: $\geq 150 \mathrm{mg} / \mathrm{dl}(1.7 \mathrm{mmol} / \mathrm{l})$;

3. Low HDL-C: $<40 \mathrm{mg} / \mathrm{dl}(1.0 \mathrm{mmol} / \mathrm{l})$ in men and $<50 \mathrm{mg} / \mathrm{dl}(1.3 \mathrm{mmol} / \mathrm{l})$ in women;

4. Hypertension: $\geq 130 / 85 \mathrm{mmHg}$;

5. Fasting hyperglycemia: $\geq 100 \mathrm{mg} / \mathrm{dl}(5.6 \mathrm{mmol} / \mathrm{l})$

\section{Coronary artery angiography}

Coronary artery angiography was performed in all patients. The procedures were performed and interpreted by trained cardiologists who had been certified as cardiologists from the Korean Society of Circulation for at least 3 years. Significant stenosis was defined by an internal diameter decrease of more than 50\%. Patients were grouped according to the number of significantly stenotic vessels into normal, 1-vessel, 2-vessel, and 3-vessel diseased groups.

\section{Statistical analysis}

Analysis was done using SPSS version 12.0 (Chicago, IL, USA). The parameters that followed normal distribution were described as mean \pm s.D. and the parameters that did not follow normal distribution were expressed as median \pm interquartile range. The normality test was performed using the KolmogorovSmirnov test. If the parameter does not follow normal distribution, log transformation (log) was performed before the statistical analyses. Bivariate correlation analyses between the variables were performed using Pearson's correlation analysis. The comparisons of the mean values for the variables and serum FABP4 levels among the different groups categorized by the number of stenotic vessels and the absence or presence of CAD, were performed using ANOVA test and Student's t-test, and the adjustment for age, BMI, gender, and fasting glucose levels were done using ANCOVA test. Multiple comparison tests were performed with post hoc analysis to compare the mean values between individual groups. The proportions of the patients with different medication history were compared using $\chi^{2}$-test. Logistic regression analysis was performed to determine whether FABP4 predicts CAD independent of age and gender. The significance was defined as a $P<0.05$.

\section{Results}

Mean age of the participants was 58.2 years and $58.5 \%$ of the participants were male (Table 1 ). Median values for serum FABP4 and fasting glucose levels were $103.0 \pm 23 \mathrm{mg} / \mathrm{dl}$ and $10.80 \pm 6.8 \mathrm{ng} / \mathrm{ml}$. Among the subjects, $31.6 \%$ had diabetes mellitus, and $46.9 \%$ had metabolic syndrome (Table 1).

Among the subjects, 135 subjects (57.7\%) had CAD with $81(34.6 \%)$ subjects having 1-vessel disease, 32 subjects (13.7\%) having 2-vessel disease, and 22 subjects $(9.4 \%)$ having 3-vessel disease (Table 1). In bivariate correlation analyses, log (FABP4) level showed no significant correlation with the variables except for age.

Mean log (FABP4) level was significantly higher in female subjects compared with male subjects $(2.68 \pm$ 0.63 vs $2.19 \pm 9.65 \mathrm{ng} / \mathrm{ml}$ ) (Table 2). There was no difference between the mean values of $\log$ (FABP4) levels according to metabolic syndrome defined by AHA/NHBLI criteria or obesity defined by $\mathrm{BMI} \geq 25 \mathrm{~kg} / \mathrm{m}^{2}$ (Table 2). Subjects with diabetes mellitus showed significantly higher mean log (FABP4) level compared with those without diabetes $(2.55 \pm 0.72$ vs $2.32 \pm$ $0.76 \mathrm{ng} / \mathrm{ml}, P=0.021$ ) (Table 2 ).

Mean log (FABP4) levels were compared according to the medication histories at the time of coronary angiograms (Table 3). Patients taking aspirin at that time showed lower mean log (FABP4) level and those taking statin and anti-hypertensives at the time of coronary angiograms showed higher mean log (FABP4) levels compared with the patients not taking those medications (Table 3). Among the anti-hypertensive

Table 1 Baseline characteristics of the subjects.

\begin{tabular}{ll}
\hline $\boldsymbol{n}=\mathbf{2 3 4}$ & Mean \pm s.D. \\
\hline Age (years) & $58.2 \pm 11.2$ \\
Male (\%) & $137(58.5)$ \\
Fasting blood glucose $(\mathrm{mg} / \mathrm{dl})$ & $103.0 \pm 23$ \\
Body mass index $\left(\mathrm{kg} / \mathrm{m}^{2}\right)$ & $25.5 \pm 2.9$ \\
Systolic blood pressure $(\mathrm{mmHg})$ & $134.4 \pm 18.7$ \\
Diastolic blood pressure (mmHg) & $84.5 \pm 10.8$ \\
Triglyceride (mg/dl) & $135.0 \pm 85$ \\
Total cholesterol (mg/dl) & $192.0 \pm 53$ \\
HDL-C (mg/dl) & $49.0 \pm 12.3$ \\
LDL-C (mg/dl) & $112.1 \pm 30.9$ \\
Serum FABP4 (ng/ml) & $10.80 \pm 6.8$ \\
Subjects with diabetes mellitus (\%) & $74(31.6)$ \\
Subjects with metabolic syndrome (\%) & $106(46.9)$ \\
Subjects with CAD (\%) & $135(57.7)$ \\
Normal vessel (\%) & $99(42.3)$ \\
1-Vessel (\%) & $81(34.6)$ \\
2-Vessel (\%) & $32(13.7)$ \\
3-Vessel (\%) & $22(9.4)$
\end{tabular}

HDL-C, high-density lipoprotein cholesterol; LDL-C, low-density lipoprotein cholesterol; FABP4, fatty acid-binding protein 4.

${ }^{a}$ The parameters that do not follow normal distribution were presented as the median \pm interquartile range, such as fasting blood glucose, triglyceride, total cholesterol, HDL-C, and serum FABP4 level.

b In eight subjects, BMI values were not available. 
Table 2 Comparisons of the mean values of log (FABP4) levels $(\mathrm{ng} / \mathrm{ml})$ according to different disease status.

\begin{tabular}{|c|c|c|c|}
\hline & \multicolumn{2}{|c|}{ Sex } & \multirow[b]{2}{*}{$P$ value } \\
\hline & Male $(n=137)$ & Female $(n=97)$ & \\
\hline $\begin{array}{l}\text { Log (FABP4) level } \\
\text { Metabolic syndrome defined by } \\
\text { AHA/NHBLI criteria }^{a}\end{array}$ & $2.19 \pm 9.65$ & $2.68 \pm 0.63$ & $<0.01$ \\
\hline $\begin{array}{l}\text { Log (FABP4) level } \\
\text { Diabetes mellitus }\end{array}$ & $\begin{array}{l}\text { Presence }(n=106) \\
2.46 \pm 0.63\end{array}$ & $\begin{array}{l}\text { Absence }(n=120) \\
2.29 \pm 0.75\end{array}$ & 0.090 \\
\hline $\begin{array}{l}\text { Log (FABP4) level } \\
\text { Obesity defined by } B M I \geq 25 \mathbf{~ k g} / \mathrm{m}^{2 a}\end{array}$ & $\begin{array}{l}\text { Presence }(n=74) \\
2.55 \pm 0.72 \\
\text { Presence }(n=128)\end{array}$ & $\begin{array}{l}\text { Absence }(n=160) \\
2.32 \pm 0.68 \\
\text { Absence }(n=98)\end{array}$ & 0.021 \\
\hline Log (FABP4) level & $2.45 \pm 0.62$ & $2.31 \pm 0.76$ & 0.120 \\
\hline
\end{tabular}

FABP4, fatty acid-binding protein 4; BMI, body mass index; log (FABP4), logarithmic transformation of serum fatty acid-binding protein 4 levels.

${ }^{a}$ In eight subjects, BMI values were not available.

medications, mean log (FABP4) levels were significantly higher in patients taking angiotensin-converting enzyme inhibitors (ACEI) or angiotensin receptor blockers (ARB) and those taking diuretics compared with patients not taking the medications (Table 3).

When the mean values of the variables were compared according to the number of stenotic vessels, subjects with CAD were older than those with normal coronary artery, and as the number of stenotic vessels increased, mean log (FABP4) levels increased (Table 4). Mean log (fasting blood glucose, FBS) significantly increased as the number of stenotic vessels increased $(P=0.001)$, and the proportion of the diabetic patients increased as the number of stenotic vessels increased from normal to 3-vessel disease (Table 4). The proportion of patients who smoked was significantly higher in those with CAD compared with those without CAD (Table 4).

Mean log (FABP4) level significantly increased as the number of stenotic vessels increased $(P=0.008)$, and in post hoc analysis, subjects with 3-vessel disease showed significantly higher mean log (FABP4) levels compared with the subjects without the $\mathrm{CAD}$ and 1-vessel disease $(P=0.001,0.003)$ (Table 4; Fig. 1), and these significances showed a strong trend toward a difference even after adjustment for age, gender, and BMI $(P=0.052)$ or, age, gender, BMI, and log (FBS) $(P=0.072)$.

When the mean log (FABP4) level was compared between the subjects with and without $\mathrm{CAD}$, subjects with CAD showed higher mean log (FABP4) level compared with the subjects without CAD with borderline significance $(P=0.096$; Table 4). Logistic regression analysis was performed with presence of $\mathrm{CAD}$ as the dependent variable, FABP4 level was not the significant determinant for CAD after adjustment for age and gender (data not shown).

\section{Discussion}

In this study, for the first time in the literature, we showed the significant association of serum FABP4 levels with CAD in humans. Mean serum FABP4 levels increased as the number of stenotic coronary arteries increased and these significances showed a strong

Table 3 Mean log (FABP4) levels according to the medication histories at the time of coronary angiograms.

\begin{tabular}{llcl}
\hline The kind of medication & & Medication history & P value \\
\hline Aspirin & Yes $(n=93,39.74 \%)$ & No $(n=144,60.26 \%)$ & 0.039 \\
& $2.28 \pm 0.71$ & $2.47 \pm 0.66$ & $<0.01$ \\
Statin & Yes $(n=56,23.93 \%)$ & $2.31 \pm 0.66$ & No $(n=91,38.89 \%)$ \\
Anti-hypertensives & $2.68 \pm 0.69$ & $2.28 \pm 0.67$ & 0.038 \\
ACEl or ARB & Yes $(n=143,61.11 \%)$ & No $(n=61,68.80 \%)$ & 0.016 \\
CCB & $2.47 \pm 0.69$ & $2.32 \pm 0.64$ & 0.222 \\
& Yes $(n=73,31.20 \%)$ & No $(n=171,73.08 \%)$ & $2.36 \pm 0.67$ \\
Diuretics & $2.55 \pm 0.75$ & No $(n=190,81.20 \%)$ & 0.041 \\
$\beta$-Blockers & Yes $(n=63,26.92 \%)$ & $2.34 \pm 0.64$ & No $(n=169,72.22 \%)$ \\
& $2.49 \pm 0.71$ & $2.37 \pm 0.69$ & 0.312 \\
\hline
\end{tabular}

Log (FABP4), logarithmic transformation of serum fatty acid-binding protein 4 levels; ACEl, angiotensin-converting enzyme inhibitor; ARB, angiotensin receptor blocker; $\mathrm{CCB}$, calcium channel blocker. 
Table 4 Comparisons of mean values of variables according to the coronary artery disease status.

\begin{tabular}{|c|c|c|c|c|c|c|}
\hline & \multirow[b]{2}{*}{$\begin{array}{l}\text { No CAD } \\
(n=99)\end{array}$} & \multirow[b]{2}{*}{$\begin{array}{l}\text { Presence of } \\
\text { CAD }(n=135)\end{array}$} & \multicolumn{3}{|c|}{ According to the number of stenotic vessels } & \multirow[b]{2}{*}{$P$ value } \\
\hline & & & $\begin{array}{l}\text { 1-vessel } \\
(n=99)\end{array}$ & $\begin{array}{l}\text { 2-vessel } \\
(n=81)\end{array}$ & $\begin{array}{l}\text { 3-vessel } \\
(n=32)\end{array}$ & \\
\hline Age (years) & $54.8 \pm 11.9^{*}$ & $60.7 \pm 10.0$ & $58.7 \pm 10.3$ & $62.5 \pm 9.4^{\star}$ & $65.2 \pm 7.9^{*}$ & $<0.01$ \\
\hline $\mathrm{DM}(\%)^{\ddagger}$ & $15(15.2)$ & $59(53.7)$ & $31(38.3)$ & $14(43.8)$ & $14(63.6)$ & $<0.01$ \\
\hline Smoking $(\%)^{\ddagger}$ & $19(19.2)$ & $48(35.56)$ & $34(42.0)$ & $7(21.9)$ & $7(31.8)$ & 0.007 \\
\hline $\log (F B S)(m g / d l)$ & $4.65 \pm 0.2^{*}$ & $4.78 \pm 0.3$ & $4.76 \pm 0.3^{\star}$ & $4.77 \pm 0.3^{\star}$ & $4.84 \pm 0.2^{*}$ & 0.001 \\
\hline $\mathrm{BMI}\left(\mathrm{kg} / \mathrm{m}^{2}\right)$ & $25.3 \pm 2.9$ & $25.7 \pm 2.8$ & $25.9 \pm 2.8$ & $26.0 \pm 2.8$ & $24.4 \pm 3.0$ & 0.139 \\
\hline $\mathrm{SBP}(\mathrm{mmHg})$ & $131.8 \pm 22.1$ & $136.3 \pm 15.5$ & $135.6 \pm 16.6$ & $134.1 \pm 12.9$ & $142.3 \pm 14.1$ & 0.103 \\
\hline $\mathrm{DBP}(\mathrm{mmHg})$ & $83.7 \pm 10.6$ & $85.1 \pm 11.0$ & $83.9 \pm 12.6$ & $85.6 \pm 8.4$ & $88.6 \pm 6.4$ & 0.228 \\
\hline $\log (T G)(m g / d l)$ & $4.88 \pm 0.5$ & $5.0 \pm 0.5$ & $4.99 \pm 0.5$ & $4.93 \pm 0.4$ & $4.96 \pm 0.4$ & 0.495 \\
\hline $\log (\mathrm{TC})(\mathrm{mg} / \mathrm{dl})$ & $5.24 \pm 0.2$ & $5.23 \pm 0.2$ & $5.22 \pm 0.2$ & $5.22 \pm 0.2$ & $5.23 \pm 0.3$ & 0.830 \\
\hline $\log (\mathrm{HDL}-\mathrm{C})(\mathrm{mg} / \mathrm{dl})$ & $3.95 \pm 0.2$ & $3.85 \pm 0.3$ & $3.85 \pm 0.4$ & $3.85 \pm 0.2$ & $3.87 \pm 0.3$ & 0.073 \\
\hline LDL-C (mg/dl) & $112.9 \pm 28.8$ & $111.5 \pm 32.4$ & $109.0 \pm 32.9$ & $114.6 \pm 31.3$ & $116.1 \pm 32.3$ & 0.696 \\
\hline Aspirin (\%) $)^{\ddagger}$ & $39(39.4)$ & $54(40.0)$ & $31(38.3)$ & $18(56.3)$ & $5(22 . \overline{7})$ & 0.095 \\
\hline Statin $(\%)^{\ddagger}$ & $8(8.1)$ & $48(35.56)$ & $25(30.9)$ & $15(46.9)$ & $8(36.4)$ & $<0.01$ \\
\hline Anti-hypertensives (\%) & $52(52.5)$ & $91(67.41)$ & $52(64.2)$ & $26(81.3)$ & $13(59.1)$ & 0.031 \\
\hline $\log (\mathrm{FABP} 4)(\mathrm{ng} / \mathrm{ml})^{\S}$ & $2.31 \pm 0.7^{*}$ & $2.46 \pm 0.6^{\prime \prime}$ & $2.34 \pm 0.6^{\dagger}$ & $2.50 \pm 0.6$ & $2.83 \pm 0.8^{*, \dagger}$ & 0.008 \\
\hline
\end{tabular}

Differences in the mean values of the parameters between the different groups were analyzed by one-way ANOVA test. $P$ values depict the significances for the trends in the differences of the mean values between the groups divided according to the number of stenotic vessels. ${ }^{\star} P<0.05$ in post hoc analysis of the subjects without CAD. ${ }^{\dagger} P<0.05$ in post hoc analysis of the subjects with 1-vessel disease. ${ }^{\mp}$ The proportions were calculated within each group divided by the number of stenotic arteries. ${ }^{\S}$ The significances shown between the log (FABP4) levels were attenuated after adjustment for age, sex, BMI $(P=0.052)$; age, sex, BMI, and log (FBS) $(P=0.072)$. " $P=0.096$ in comparison analysis of the subjects without CAD analyzed with Student's $t$-test. CAD, coronary artery disease; DM, diabetes mellitus; FBS, fasting blood sugar; BMI, body mass index; SBP, systolic blood pressure; DBP, diastolic blood pressure; TG, triglyceride; TC, total cholesterol; HDL-C, high-density cholesterol; LDL-C, low-density cholesterol; FABP4, fatty acid-binding protein 4; log (FABP4), logarithmic transformation of serum FABP4 levels; log (FBS), logarithmic transformation of fasting glucose levels.

tendency for a difference even after adjustments were made with age, gender, BMI, and fasting blood glucose. Serum FABP4 levels showed no association with metabolic syndrome or obesity categorized by BMI values. The attenuation of the significant associations between serum FABP4 levels and the number of stenotic coronary arteries after adjustment for age and fasting blood glucose, suggests that the effect of circulating FABP4 levels on atherosclerosis might be explained through the aging process and impaired glucose metabolism. Another interesting point of our study was that serum FABP4 levels showed significant differences in subjects with different medication, including aspirin, statin, and anti-hypertensive drugs.

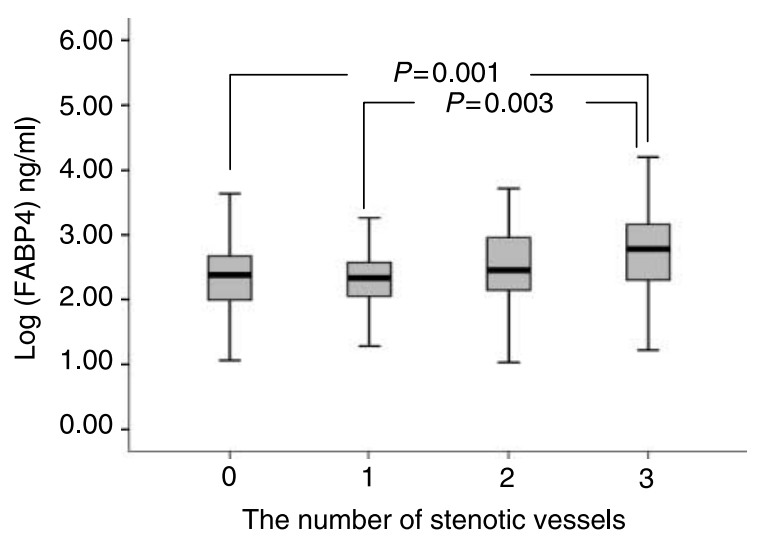

Figure 1 The comparison of the log (FABP4) according to the number of stenotic coronary arteries. Log (FABP4), logarithmic transformation of fatty acid-binding protein 4 .
From the fact that FABP4 is expressed in adipocyte and macrophages $(6,14)$, and the high level of FABP4 was found to be expressed in atherosclerotic lesions in mice and humans (22), and the fact that metabolic syndrome and diabetes is considered to be important risk factors for atherosclerosis, the possibility of the role of FABP4 in the development of atherosclerosis was investigated in clinical studies. Tuncman et al. successfully produced genetic support for the involvement of FABP4 in atherosclerosis in humans (17). They identified a genetic variant $(\mathrm{T}-87 \mathrm{C})$ at the promoter region of FABP4 in humans resulted in decreased adipose tissue FABP4 expression due to alteration of the CAAT box/enhancer-binding protein binding and reduced transcriptional activity of FABP4 promoter and they validated these genetic mutation in 7899 participants, that the carriers of this polymorphisms showed lower serum triglyceride levels and significantly reduced risk for coronary heart disease and type 2 diabetes compared with wild-type allele. Furuhashi et al. (13) demonstrated more functionally confirmative results in that the treatment of $\mathrm{ApoE}^{-I-}$ mouse model with a FABP4 inhibitor resulted in protection against atherosclerosis and metabolic syndrome, suggesting that the pharmacologic intervention for FABP4 inhibition might also show beneficial effects against diabetes and cardiovascular disease. Recent work from Yeung et al. showed positive correlation of serum FABP4 levels with carotid atherosclerosis in Chinese women, suggesting another clinical evidence for the role of FABP4 in the development of atherosclerosis in humans (18). However, no studies have been reported on the direct 
association between serum FABP4 levels and CAD in human.

In this study, the number of stenotic coronary arteries was significantly associated with increasing circulating concentrations of serum FABP4 levels, suggesting the involvement of FABP4 in the development of atherosclerosis. Subjects with the highest number of stenotic vessels showed significantly higher serum FABP4 levels compared with those with 1-vessel and those without CAD, suggesting the enlarging influence of FABP 4 levels as the extent of stenosis progressed. The mechanism for the correlation of serum FABP4 levels with coronary artery stenosis might be explained in several ways. Adenovirus-mediated overexpression of FABP4 in human macrophages directly induces foam cell formation by increasing intracellular cholesterol ester accumulation (22). On the contrary, ablation of FABP4 expression in macrophage increases cholesterol efflux and prevents oxidized LDL-induced foam cell formation. Apolipoprotein E (ApoE) deficient mice also deficient in FABP4 showed protection from atherosclerosis in the absence of significant differences in serum lipids or insulin sensitivity, and $\mathrm{ApoE}^{-1-}$ mice with $\mathrm{FABP} 4^{-/+}$adipocytes and $\mathrm{FABP} 4^{-/-}$macrophages showed comparable reduction in atherosclerotic lesions to those with total FABP4 deficiency, indicating an independent role for macrophage FABP 4 in atherogenesis (12). Since FABP4 was traditionally thought to be a cytoplasmic protein, whether the circulating FABP4 might have equal influence and have the same pathologic effects on vasculature in cellular level, has to be clarified with more experimental evidences. Further studies connecting the serum FABP4 and circulating inflammatory markers of macrophage function and activity might uncover interesting results relating the effects of serum FABP4 on vasculature in humans.

In this study, the association observed between coronary artery stenosis and serum FABP4 were attenuated after adjustment for age and fasting blood glucose. This attenuation suggests that the effect of circulating FABP4 on CAD might be through the differences in age and the serum concentration of fasting blood glucose levels in different groups of coronary artery stenosis. Serum FABP4 levels have been shown to be positively correlated with glucose levels and insulin levels $(14,16)$, and in the prospective study by Tso et al. elevated baseline serum FABP 4 level predicted the development of type 2 diabetes (15). Subjects with impaired glucose metabolism or diabetes have higher serum FABP4 levels than those with normal glucose metabolism (18), and subjects having genetic polymorphism in FABP4 promoter, showed significantly lower risk for developing type 2 diabetes mellitus (17). Similar results were shown in our study population, in that subjects with diabetes had significantly higher serum levels of FABP4 compared with those without diabetes, and more proportion of diabetic patients were present in the group with more number of stenotic arteries compared with the groups with lesser number of stenotic vessels. Such adverse effects of FABP4 on glucose metabolism must have influenced the association of serum FABP4 with coronary artery stenosis in this study, as the significances were attenuated after adjustment for fasting blood glucose.

Although the precise mechanisms explaining the role of FABP4 in glucose metabolism is not yet fully understood, the role FABP4 as the link for the interplay between macrophage and adipocyte, could be one of the explanations for the action of this molecule in glucose metabolism and inflammation $(9,13,23-25)$. In a very recent interesting work by Furuhashi et al. (13), selective deletion of FABPs in adipocytes resulted in reduced expression of inflammatory cytokines in macrophages, whereas the same deletion in macrophages led to enhanced insulin signaling and glucose uptake in adipocytes, suggesting that the interaction between these two cells affect systemic insulin sensitivity and glucose metabolism, especially in adipose tissues. Although these observations in animal studies could be replicated in human body needs further investigation, it is clear from our study result that serum FABP 4 has significant association with coronary atherosclerosis, and must have been affected by the differences in the glucose metabolism in subjects with different degrees of atherosclerosis.

Another interesting result of our study was that serum FABP4 levels showed significant differences according to the different medication histories, including aspirin, statin and various anti-hypertensive medications, all of which could have affected the atherosclerotic change of the coronary vessels of the participants. As could be expected from the known antiatherogenic effects of aspirin (26), the subjects who were taking aspirin at the time of coronary angiogram showed significantly lower serum FABP4 levels compared with those who were not. The subjects who were taking statin or anti-hypertensives showed significantly higher serum FABP4 levels compared with those who were not taking the medications. Although our result contradicts a previous study that suggested the effect of atorvastatin on the prevention of the rise of FABP4 expression caused by oxidized LDL in macrophage (27), as the exact duration of past medial histories of the participants for hypertension or hyperlipidemia were not available, we could speculate that, the subjects who were taking statins and antihypertensive medications might have suffered from the particular diseases and thus, so have the vessels, showing the progression of atherosclerosis, and the subsequent rise in the serum FABP4 levels. Among the anti-hypertensive medications, subjects who were taking ACEI or ARB, so-called renin-angiotensin system (RAS) blockers, showed significantly higher serum FABP4 levels compared with the subjects who were not. Although there are few studies regarding the effects of ARBs on the improvement of steatohepatitis and 
inflammation in liver in rat models $(28,29)$, the direct effect of RAS blockade on FABP4 action needs further clarification. Taken together, the effects of multiple antiatherogenic interventions on serum FABP4 levels might be another interesting field to be discovered in the future.

Although a direct causal relationship between serum FABP4 and coronary artery stenosis could not be drawn due to the cross-sectional nature of the study, this was the first study to report the association between serum FABP 4 levels and coronary artery stenosis in humans. A more well-designed prospective study in an extended population with additional measurements of more specified biomarkers related with vascular inflammation would give more clues to clarify the mechanisms related with the clear link between serum FABP4 and atherosclerosis in humans.

\section{Declaration of interest}

All the authors of this manuscript declare that there is no conflict of interest that could be perceived as prejudicing the impartiality of the research reported.

\section{Funding}

This research did not receive any specific grant from any funding agency in the public, commercial or not-for-profit sector.

\section{Acknowledgements}

We want to give special thanks to Prof Sun-Woo Kim and Sung-Woo Park in the Department of Endocrinology and Metabolism, and Jin-Ho Kang and Man-Ho Lee in the Department of Cardiology, Kangbuk Samsung Hospital Sungkyunkwan University School, Seoul, Korea for their limitless support, both scientifically and psychologically.

The results of this paper was presented as a poster at the 44th annual meeting of European Association for the Study of Diabetes held in Rome, Italy in September, 2008.

\section{References}

1 Yoon KH, Lee JH, Kim JW, Cho JH, Choi YH, Ko SH, Zimmet P \& Son HY. Epidemic obesity and type 2 diabetes in Asia. Lancet 2006 368 1681-1688.

2 American Heart Association. Heart Disease and Stroke Statistics 2005 Update Dallas, TX: American Heart Association, 2005.

3 Poirier P. Recurrent cardiovascular events in contemporary cardiology: obesity patients should not rest in PEACE. European Heart Journal 200627 1390-1391.

4 Despres JP. Is visceral obesity the cause of the metabolic syndrome? Annals of Medicine 200638 52-63.

5 Fortuno A, Rodriguez A, Gomez-Ambrosi J, Fruhbeck G \& Diez J. Adipose tissue as an endocrine organ: role of leptin and adiponectin in the pathogenesis of cardiovascular diseases. Journal of Physiology and Biochemistry 200359 51-60.

6 Makowski L \& Hotamisligil GS. Fatty acid binding proteins-the evolutionary crossroads of inflammatory and metabolic responses. Journal of Nutrition 20041342464 S-2468S.

7 Haunerland NH \& Spener F. Fatty acid-binding proteins - insights from genetic manipulations. Progress in Lipid Research 200443 328-349.
8 Chmurzyńska A. The multigene family of fatty acid-binding proteins (FABPs): function, structure and polymorphism. Journal of Applied Genetics 200647 39-48.

9 Maeda K, Cao H, Kono K, Gorgun CZ, Furuhashi M, Uysal KT, Cao Q, Atsumi G, Malone H, Krishnan B, Minokoshi Y, Kahn BB, Parker RA \& Hotamisligil GS. Adipocyte/macrophage fatty acid binding proteins control integrated metabolic responses in obesity and diabetes. Cell Metabolism 20051 107-119.

10 Hotamisligil GS, Johnson RS, Distel RJ, Ellis R, Papaioannou VE \& Spiegelman BM. Uncoupling of obesity from insulin resistance through a targeted mutation in aP2, the adipocyte fatty acid binding protein. Science $1996 \mathbf{2 7 4} 1377-1379$.

11 Uysal KT, Scheja L, Wiesbrock SM, Bonner-Weir S \& Hotamisligil GS. Improved glucose and lipid metabolism in genetically obese mice lacking aP2. Endocrinology $20001413388-3396$.

12 Makowski L, Boord JB, Maeda K, Babaev VR, Uysal KT, Morgan MA, Parker RA, Suttles J, Fazio S, Hotamisligil GS \& Linton MF. Lack of macrophage fatty-acid-binding protein aP2 protects mice deficient in apolipoprotein $\mathrm{E}$ against atherosclerosis. Nature Medicine 20017 699-705.

13 Furuhashi M, Tuncman G, Görgün CZ, Makowski L, Atsumi G, Vaillancourt E, Kono K, Babaev VR, Fazio S, Linton MF, Sulsky R, Robl JA, Parker RA \& Hotamisligil GS. Treatment of diabetes and atherosclerosis by inhibiting fatty-acid-binding protein aP2. Nature 200721 959-965.

$14 \mathrm{Xu} \mathrm{A}$, Wang Y, Xu JY, Stejskal D, Tam S, Zhang J, Wat NMS, Wong WK \& Lam KSL. Adipocyte fatty-acid binding protein is a plasma biomarker closely associated with obesity and metabolic syndrome. Clinical Chemistry 200652 405-413.

15 Tso AW, Xu A, Sham PC, Wat NM, Wang Y, Fong CH, Cheung BM, Janus ED \& Lam KS. Serum adipocyte fatty acid binding protein as a new biomarker predicting the development of type 2 diabetes: a 10-year prospective study in a Chinese cohort. Diabetes Care 2007 $302667-2672$.

$16 \mathrm{Xu}$ A, Tso AW, Cheung BM, Wang Y, Wat NM, Fong CH, Yeung DC, Janus ED, Sham PC \& Lam KS. Circulating adipocyte-fatty acid binding protein levels predict the development of the metabolic syndrome: a 5-year prospective study. Circulation $2007 \mathbf{1 1 5}$ 1537-1543.

17 Tuncman G, Erbay E, Hom X, De Vivo I, Campos H, Rimm EB \& Hotamisligil GS. A genetic variant at the fatty acid-binding protein aP2 locus reduces the risk for hypertriglyceridemia, type 2 diabetes, and cardiovascular disease. PNAS 2006103 6970-6975.

18 Yeung DCY, Cheung CWS, Wat NMS, Yau MH, Fong CHY, Chau MT \& Lam KSL. Serum adipocyte fatty acid-binding protein levels were independently associated with carotid atherosclerosis. Arteriosclerosis, Thrombosis, and Vascular Biology $2007 \mathbf{2 7}$ 1796-1802.

19 American Diabetes Association. Standards of medical care in diabetes. Diabetes Care 200730 S4-S41.

20 Grundy SM, Cleeman JI, Daniels SR, Donato KA, Eckel RH, Franklin BA, Gordon DJ, Krauss RM, Savage PJ, Smith SC Jr, Spertus JA \& Costa F. American Heart Association; National Heart, Lung, and Blood Institute. Diagnosis and management of the metabolic syndrome: an American Heart Association/National Heart, Lung, and Blood Institute Scientific Statement. Circulation $20051122735-2752$.

21 WHO Western Pacific Region, IASO and IOTF: the Asia-Pacific Perspective: redefining obesity and its treatment. Sydney, Australia, Health Communications Australia Pty Limit, 2000.

$22 \mathrm{Fu} \mathrm{Y,} \mathrm{Luo} \mathrm{N,} \mathrm{Lopes-Virella} \mathrm{MF} \mathrm{\&} \mathrm{Garvey} \mathrm{WT.} \mathrm{The} \mathrm{adipocyte}$ lipid binding protein (ALBP/aP2) gene facilitates foam cell formation in human THP-1 macrophages. Atherosclerosis 2002 $165259-269$.

23 Coe NR, Simpson MA \& Bernlohr DA. Targeted disruption of the adipocyte lipid-binding protein (aP2 protein) gene impairs fat cell lipolysis and increases cellular fatty acid levels. Journal of Lipid Research 199940 967-972. 
24 Scheja L, Makowski L, Uysal KT, Wiesbrock SM, Shimshek DR, Meyers DS, Morgan M, Parker RA \& Hotamisligil GS. Altered insulin secretion associated with reduced lipolytic efficiency in aP2 - / - mice. Diabetes 199948 1987-1994.

25 Fisher RM, Eriksson P, Hoffstedt J, Hotamisligil GS, Thörne A, Rydén M, Hamsten A \& Arner P. Fatty acid binding protein expression in different adipose tissue depots from lean and obese individuals. Diabetologia 200144 1268-1273.

26 Yasuda O, Takemura Y, Kawamoto H \& Rakugi H. Aspirin: recent developments. Cellular and Molecular Life Sciences 200865 354-358.

27 Llaverias G, Noé V, Peñuelas S, Vázquez-Carrera M, Sánchez RM, Laguna JC, Ciudad CJ \& Alegret M. Atorvastatin reduces CD68, FABP4, and HBP expression in oxLDL-treated human macrophages. Biochemical and Biophysical Research Communications 2004 318 265-274.
28 Kitamura K, Tada S, Nakamoto N, Toda K, Horikawa H, Kurita S, Tsunematsu S, Kumagai N, Ishii H, Saito H \& Hibi T. Rho/Rho kinase is a key enzyme system involved in the angiotensin II signaling pathway of liver fibrosis and steatosis. Journal of Gastroenterology and Hepatology 200722 2022-2033.

29 Kurita S, Takamura T, Ota T, Matsuzawa-Nagata N, Kita Y, Uno M, Nabemoto S, Ishikura K, Misu H, Ando H, Zen Y, Nakanuma Y \& Kaneko S. Olmesartan ameliorates a dietary rat model of nonalcoholic steatohepatitis through its pleiotropic effects. European Journal of Pharmacology $2008 \mathbf{5 8 8} 316-324$.

Received 26 October 2008

Accepted 8 November 2008 